\title{
Tuberculosis espinal: opciones de tratamiento mas allá de las guías clínicas
}

Spinal tuberculosis - Treatment options beyond guidelines

Inês Esteves Cruz, João Mourato Torres

Hospital Egas Moniz - CHLO, Lisboa, Portugal

\begin{abstract}
Spinal tuberculosis, a presentation of extrapulmonary tuberculosis, usually presents with chronic back pain or deformity. The diagnosis is often delayed due to the insidious and nonspecific nature of the initial symptoms. The thoracic spine is more commonly affected, but it can affect other segments or be multisegmental. The site and extent of the lesion will determine the resulting neurological deficits which can lead to significant morbidity and functional impairment. Combination chemotherapy for 6 months is recommended, but some experts may recommend therapy for 9 to 12 months depending on the circumstances. Adjunct corticotherapy and surgery are also options in specific cases. Further studies will be needed in order to better understand the role of these therapies in severe tuberculosis, and in particular spinal tuberculosis.
\end{abstract}

Keywords: Spinal tuberculosis; Spinal cord compression; Paraplegia; Corticosteroid therapy; Mycobacterium tuberculosis.

Tuberculosis (TB) is a multisystemic disease that mainly affects the lungs, but can involve other organs and systems. According to the World Health Organization (WHO), extrapulmonary TB has a prevalence of up to $20-25 \%$. The most frequent sites other than the lungs are the lymphatic system, pleura and bone ${ }^{1}$. Extrapulmonary TB can present with signs and symptoms related to the affected organ system or as a chronic systemic inflammation, namely with fever, night sweats, asthenia, weight loss or cachexia. The presentation can be insidious, which often undermines and delays the correct diagnosis.

We present a case of a 21-year-old woman, with no relevant previous medical history, that reported loss of strength and sensation in the lower limbs that slowly progressed to the upper extremities over a period of 2 years. The patient also reported neuropathic pain in the cervical region, anorexia, asthenia and a vespertine fever. She had been living in Guinea-Bissau and was sent to Portugal to seek further medical care. The neurological examination revealed a spastic paraplegia, with only mild decrease in muscular strength in the upper extremities (4/5) and loss of pain and light-touch sensation as well as deep sensation in the lower limbs. Increased stretch reflexes in the lower extremities and a positive bilateral Babinsky sign were present. The clinical examination also revealed an enlarged and painless cervical lymph node.

Magnetic Resonance Imaging (MRI) revealed a retropharyngeal abscess (C1-C5) extending to the anterior epidural space at $\mathrm{C} 4-\mathrm{C5}$ and compressing the spinal cord at this level [Fig. 1]. In the dorso-lumbar region, a severe deformity of the body of D7 was apparent. In addition, a pre- and paravertebral abscess between D6-D8 was found, extending to the anterior epidural space and into the left intra-thoracic region [Fig. 2]. Biopsies of the cervical lymph node, retropharyngeal and intra-thoracic
Fig. 1. Cervical MRI showing retropharyngeal abscess (C1-C5) extending to the anterior epidural space at C4-C5

and compressing the spinal cord at this level.

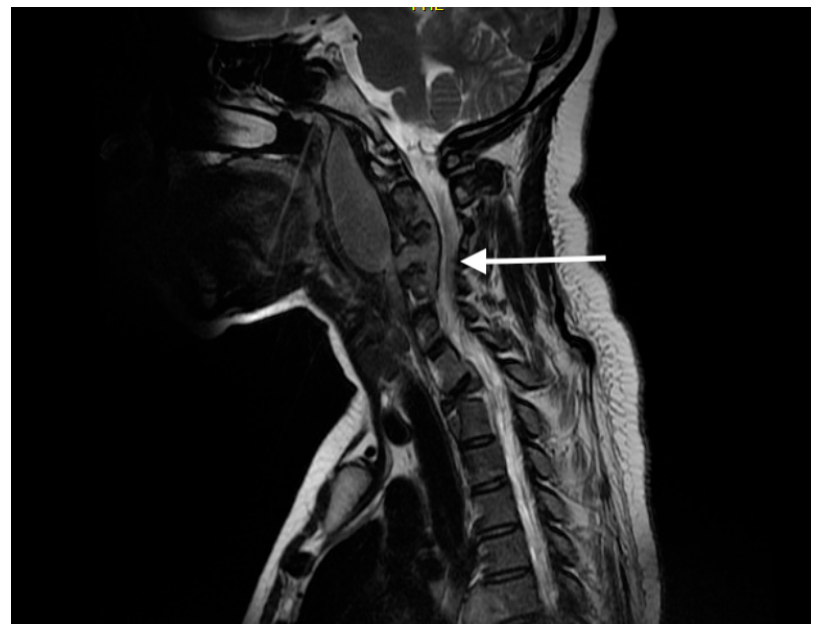

Fig. 2. Dorso-lumbar MRI demonstrates a severe deformity of the body of $D 7$, pre- and paravertebral abscess between D6-D8, extending to the anterior epidural space.

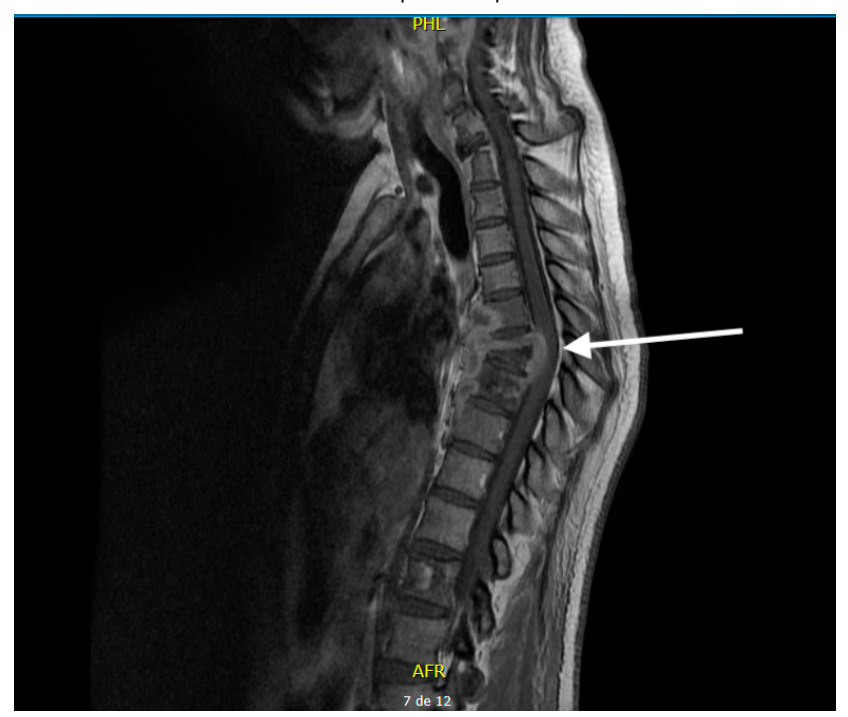


abscesses were performed. Ziehl-Neelsen staining for acidfast bacilli was negative in all, but polymerase chain reaction (PCR) - based assay was positive for Mycobacterium tuberculosis complex in intra-thoracic abscess.

The patient was thus started on isoniazid (300 mg), rifampin (600 mg), pyrazinamide (1500 mg) and ethambutol (1200 mg) (HRZE). After two weeks without clinical improvement, and given the severity of the neurologic deficits, treatment with prednisone $1 \mathrm{mg} / \mathrm{Kg} /$ day (60 $\mathrm{mg} /$ day) was instituted. Finally, after 3 months of therapy with HRZE and 8 weeks of prednisone (2 weeks full dose with tapering over a period of 6 weeks) the patient began to recover. At the time of discharge, she was able to walk without the need of any assistive device and was independent in all other activities of daily living. In view of the considerable outspread of the infection, treatment with isoniazid and rifampin was extended for 12 months.

Spinal tuberculosis usually presents with chronic back pain or deformity, but the diagnosis is often delayed due to the insidious and nonspecific nature of the initial symptoms. The thoracic spine is more commonly affected, but it can affect other segments or be multisegmental. The site and extent of the lesion will determine the resulting neurological deficits that can lead to significant morbidity and functional impairment. Combination chemotherapy for 6 months is recommended, but some experts may recommend therapy for 9 to 12 months depending on the circumstances ${ }^{2,3}$. Adjunct corticotherapy and surgery are also options in specific cas$\mathrm{es}^{2,3,4}$. Further studies will be needed in order to better understand the role of these therapies in severe tuberculosis, and in particular spinal tuberculosis.

\section{REFERENCES}

1. World Health Organization, Stop TB Initiative (World Health Organization. Treatment of tuberculosis: guidelines. Geneva: World Health Organization; 2010.

2. Nahid P, Dorman S, Alipanah N, Barry P, Brozek J, Cattamanchi A et al. Official American Thoracic Society/Centers for Disease Control and Prevention/Infectious Diseases Society of America clinical practice guidelines: Treatment of drugsusceptible tuberculosis. Clinical Infectious Diseases. 2016; 63(7):e147-95.

3. Internal Clinical Guidelines Team (UK). Tuberculosis: Prevention, Diagnosis, Management and Service Organisation. London: National Institute for Health and Care Excellence (UK); 2016 Jan. (NICE Guideline, No. 33.)

4. Guidelines for treatment of drug-susceptible tuberculosis and patient care, 2017 update. Geneva: World Health Organization; 2017. Licence: CC BY-NC-SA 3.0 IGO. 